\title{
Targeted High-Resolution Taxonomic Identification of Bifidobacterium longum subsp. infantis Using Human Milk Oligosaccharide Metabolizing Genes
}

\author{
Lauren Tso ${ }^{\dagger}$, Kevin S. Bonham ${ }^{+}(\mathbb{D}$, Alyssa Fishbein, Sophie Rowland and Vanja Klepac-Ceraj *(D) \\ Department of Biological Sciences, Wellesley College, Wellesley, MA 02481, USA; ltso@wellesley.edu (L.T.); \\ kbonham@wellesley.edu (K.S.B.); afishbein@wellesley.edu (A.F.); srowland@wellesley.edu (S.R.) \\ * Correspondence: vklepacc@wellesley.edu \\ † These authors contributed equally to this work.
}

Citation: Tso, L.; Bonham, K.S.;

Fishbein, A.; Rowland, S.;

Klepac-Ceraj, V. Targeted

High-Resolution Taxonomic

Identification of Bifidobacterium

longum subsp. infantis Using Human Milk Oligosaccharide Metabolizing

Genes. Nutrients 2021, 13, 2833.

https://doi.org/10.3390/nu13082833

Received: 7 July 2021

Accepted: 8 August 2021

Published: 18 August 2021

Publisher's Note: MDPI stays neutral with regard to jurisdictional claims in published maps and institutional affiliations.

Copyright: (c) 2021 by the authors. Licensee MDPI, Basel, Switzerland. This article is an open access article distributed under the terms and conditions of the Creative Commons Attribution (CC BY) license (https:// creativecommons.org/licenses/by/ $4.0 /)$.
Abstract: Bifidobacterium longum subsp. infantis (B. infantis) is one of a few microorganisms capable of metabolizing human breast milk and is a pioneer colonizer in the guts of breastfed infants. One current challenge is differentiating $B$. infantis from its close relatives, B. longum and B. suis. All three organisms are classified in the same species group but only $B$. infantis can metabolize human milk oligosaccharides (HMOs). We compared HMO-metabolizing genes across different Bifidobacterium genomes and developed $B$. infantis-specific primers to determine if the genes alone or the primers can be used to quickly characterize $B$. infantis. We showed that $B$. infantis is uniquely identified by the presence of five HMO-metabolizing gene clusters, tested for its prevalence in infant gut metagenomes, and validated the results using the B. infantis-specific primers. We observed that only 15 of $203(7.4 \%)$ children under 2 years old from a cohort of US children harbored B. infantis. These results highlight the importance of developing and improving approaches to identify B. infantis. A more accurate characterization may provide insights into regional differences of $B$. infantis prevalence in infant gut microbiota.

Keywords: human milk oligosaccharide metabolizing genes; Bifidobacterium infantis; metagenomics; comparative genomics; child development; pediatric cohort; metabolic potential

\section{Introduction}

Human microbiota has coevolved with humans for tens of thousands of years [1]. This coexistence requires a tight partnership between the host and its resident microorganisms. For example, the abundant oligosaccharides found in human milk cannot be digested by the infant who feeds on them. Instead, these are used to support the growth of the pioneer gut colonizers, who then, in turn, support the development of the infant $[2,3]$. In particular, taxa in the Bifidobacterium genus often have genes that allow them to metabolize the complex carbohydrates in their hosts [4-6]. The subspecies Bifidobacterium longum subsp. infantis (B. infantis), for example, can import and degrade a variety of complex human milk oligosaccharides (HMOs) [7]. Organisms belonging to the same longum subgroup, B. longum subsp. suis (B. suis) and B. longum subsp. longum (B. longum), are unable to metabolize HMOs.

The functional potential of $B$. infantis to metabolize HMOs lies in five genomic loci dedicated to the import of HMOs and their processing [8]. The 79 genes within these five clusters reveal substantial glycolytic potential [8], allowing for the transmembrane import of milk oligosaccharides into the cytosol and later cleaving into monosaccharides [9]. The proximity of these genes indicates a role in the regulation of a common metabolic pathway that has coevolved with milk components to outcompete other microbes in the infant gut [8]. In the developing infant gut, where milk is the primary source of nutrients, this ability provides a selective advantage [9]. Given that other taxa of interest may also 
have differential functional potential, high-resolution identification of all species in the community is desirable. One of the most widely used culture-independent methods to taxonomically profile gut microbiomes, $16 \mathrm{~S}$ rRNA amplicon sequencing, cannot differentiate $B$. infantis from its closest relatives [10-13]. Whole metagenome ("shotgun") sequencing can provide additional taxonomic resolution, but existing tools for assembly-free taxonomic assignment also commonly fail to differentiate subspecies [14,15] infantis from other B. longum subspecies. It remains unclear whether this $\mathrm{HMO}$ metabolic potential is confined to a single lineage or is shared across closely related organisms (e.g., through horizontal gene transfer). The origins of these gene cassettes also remain obscure, though other species such as species belonging to Bifidobacterium, Lactobacillus, and Bacteroides genera have HMO use potential $[9,16-21]$. Understanding the distribution of these genes in microbial species may enable using metagenomic sequencing for the interrogation of $B$. infantis colonization, succession, and distribution in the infant microbiome in new stool metagenomes as well as those that are already available [9]. In this study, we tested the in vitro and in silico use of these 79 HMO-metabolizing genes and determined that they alone can identify $B$. infantis in a stool sample and assess its prevalence in the population. We then applied this characterization of $B$. infantis to search for its presence in a US pediatric cohort with shotgun metagenomic sequencing. We validated these results by designing and benchmarking subspecies-specific PCR primers targeting a subset of these HMO-metabolizing genes.

\section{Materials and Methods}

\subsection{Bacterial Genomes}

The genome sequences of 387 Bifidobacterium species and subspecies were downloaded in FASTA format from the National Center for Biotechnology Information (NCBI) database. Genomes included 4 Bifidobacterium breve and 383 strains belonging to Bifidobacterium longum group, spanning all three subspecies (43 B. infantis, 167 B. longum, 3 B. suis). In addition, 170 of the genomes were unspecified $B$. longum group strains and were also included in the analysis (Supplementary Materials Table S1).

\subsection{Calculation of ANI Values}

All pairwise, whole-genome sequence comparisons were performed using average nucleotide identity (ANI). ANI is an in silico substitute for DNA-DNA hybridization (DDH) and thus is useful for delineating species boundaries [22]. The Python module pyani (v 0.2.7) was used to calculate ANI values [23]. Specifically, the genomic sequence from one of the genomes in a pair was aligned against the other using the matching algorithm MUMmer (v 4.0.0) through the ANIm command [24]. The calculated ANI value was the average percentage nucleotide identity for all matching regions between a pair of genomes. A percentage identity table returned from this analysis was then converted to a distance matrix using R 3.6.0 (Supplementary Tables S2 and S3). The neighbor-joining ANI tree consisting of 387 Bifidobacterium genomes was built using the default parameters with the phytools (v 0.6-99) package. Each tree was rooted by the four B. breve genomes included as an outgroup and bootstrapped with values assigned to internal edges over 100 iterations.

\subsection{Genomic Analysis of HMO-Metabolizing Clusters}

Specificity of the HMO-metabolizing gene cluster was determined in published genomes from NCBI and sequenced metagenomes from fecal samples. The presence of HMO-metabolizing clusters in each of the Bifidobacterium genomes from NCBI was determined by BLASTN hits and filtered for $95 \%$ identity. Heatmaps were colored by the presence of at least one BLAST hit to a gene. The presence of HMO-metabolizing clusters in the non-redundant database from NCBI was determined by BLASTP hits and filtered for $80 \%$ identity [25].

Evolutionary pressures of the 78 protein-coding HMO-metabolizing genes were assessed by differences in the substitution rates at nonsynonymous $(\mathrm{dN})$ and synonymous 
sites (dS) using MEGA X (v 10.1.8) [26,27]. A significant difference in the relative abundance of synonymous and nonsynonymous substitutions implies non-neutral selection, with a higher ratio of nonsynonymous substitutions indicating positive selection and a lower ratio indicating purifying selection [28].

\subsection{Subjects and Samples}

Samples $(n=1092)$ used in this study were collected from the RESONANCE Cohort (Providence, RI), an accelerated-longitudinal study of healthy infants and children. Children in the cohort $(n=583)$ were between 33 days and 15 years old (mean $=4.47$ years), with 144 samples collected from children less than 1 year old, and 205 from children under 2 years old (Supplementary Materials Table S4). Approximately 70\% of subjects were born vaginally, and approximately $80 \%$ were fed at least some breastmilk. All procedures and experiments in the study were approved by and followed the guidelines of the local review board. Written consent was obtained from all parents or guardians of participants. Fecal samples were collected by patients in OMR-200 tubes (OMNIgene GUT, DNA Genotek, Ottawa, ON, Canada), brought to the RI lab and frozen within $24 \mathrm{~h}$ at $-80^{\circ} \mathrm{C}$ in the RI lab.

\subsection{Sample Processing and Sequencing}

All samples were processed at Wellesley College (Wellesley, MA, USA). Nucleic acids were extracted from stool samples using the RNeasy PowerMicrobiome kit automated on the QIAcube (Qiagen, Germantown, MD, USA), excluding the DNA degradation steps. Extracted DNA was sequenced using shotgun metagenomic sequencing at the Integrated Microbiome Resource (IMR, Dalhousie University, Halifax, NS, Canada). A pooled library (max 96 samples per run) was prepared using the Illumina Nextera Flex Kit for MiSeq and NextSeq (a PCR-based library preparation procedure) from $1 \mathrm{ng}$ of each sample where samples were enzymatically sheared and tagged with adaptors, PCR amplified while adding barcodes, purified using columns or beads, and normalized using Illumina beads or manually. Samples were then pooled onto a plate and sequenced on the Illumina NextSeq 550 platform using $150+150$ bp paired-end "high output" chemistry, generating 400 million raw reads and $120 \mathrm{~Gb}$ of sequence. The list of samples used in this study is listed in Supplementary Materials Table S5. All metagenomic data were deposited in SRA in BioProject PRJNA695570.

\subsection{Identification of B. infantis in Metagenomic Samples by the Presence of HMO-Metabolizing Clusters}

The presence of $B$. infantis in each of the 1092 sample metagenomes included in our study was determined by that of the majority (75\%) of the $79 \mathrm{HMO}$-metabolizing genes in the gene cluster. Metagenomic samples were analyzed using the bioBakery [29] family of tools with default parameters. Briefly, KneadData (v 0.7.1) was used to trim and filter raw sequence reads and to separate human reads from bacterial sequences. Samples that passed quality control were taxonomically profiled to the species level using MetaPhlAn (v 3.0.1). Paired-end reads of the $150 \mathrm{bp}$ nucleotide sequences acted as inputs against a reference database including the $79 \mathrm{HMO}-$ metabolizing genes using the alignment tool Bowtie2 (v 2.4.2) [30]. Bowtie2 was used in these searches, and not in any others, for its specific ability in aligning short reads to relatively long genomes. The presence of a gene was noted if at least one mapped alignment was found to that gene.

\subsection{Primer Design and Testing}

Previously, published primers designed to target $B$. infantis were either too specific or did not target $B$. infantis or were too broad and targeted other members of the B. longum group (Table $1 ;[2,31,32]$ ). We decided to test the existing primers in silico and to develop new primer sets. To find candidate regions, we analyzed 79 genes associated with the five HMO-metabolizing clusters and selected genes that were present in all B. infantis and that had gene sections highly conserved across the different strains. 
Table 1. Primers targeting HMO metabolizing and 16S rRNA genes.

\begin{tabular}{|c|c|c|c|c|c|c|c|}
\hline Gene & Target Organism & Name & Sequence $\left(5^{\prime}-3^{\prime}\right)$ & $\begin{array}{l}\text { Annealing } \\
\text { Temp }\left({ }^{\circ} \mathrm{C}\right)\end{array}$ & $\%$ Coverage ${ }^{1}$ & References & Length $(b p)^{2}$ \\
\hline \multirow[t]{2}{*}{$\begin{array}{l}\text { exo- } \alpha \text {-sialidase } \\
\text { (Blon_2348) }\end{array}$} & B. longum infantis & Sia-266F & GACGAGGAGGAATACAGCAG & 58 & 100 & This study & 410 \\
\hline & & Sia-676R & CACGAACAGCGAATCATGGATT & & & & \\
\hline \multirow[t]{2}{*}{$\begin{array}{l}\text { glycoside-hydrolase } \\
\text { (Blon_2355) }\end{array}$} & B. longum infantis & GH-750F & GCGCCATCCTGGTGATGTTATT & 59 & 100 & This study & 498 \\
\hline & & GH-1248R & CTACGTGATCTGGGAGAGTTTC & & & & \\
\hline \multirow{2}{*}{$\begin{array}{l}\text { haloacid dehalogenase } \\
\text { domain protein hydrolase } \\
\text { (Blon_2356) }\end{array}$} & B. longum infantis & HH-60F & CCACAATGTCATCGACCATCTG & 59 & 83 & This study & 474 \\
\hline & & $\mathrm{HH}-534 \mathrm{R}$ & CCGAAGTATTCGGATGCCTATG & & & & \\
\hline \multirow[t]{2}{*}{$\begin{array}{l}\text { glycoside hydrolase } \\
\text { (Blon_2358) }\end{array}$} & B. longum infantis & GH-492F & CGATGATGTGCTGGATTCGTTC & 58 & 74 & This study & 510 \\
\hline & & GH-1002R & CTCGACCATTCCAAGATGCTCA & & & & \\
\hline \multirow[t]{2}{*}{ Sialidase } & B. longum infantis & Inf $2348-F$ & ATACAGCAGAACCTTGGCCT & 60 & 50 & [32] & 280 \\
\hline & & Inf 2348-R & GCGATCACATGGACGAGAAC & & & & \\
\hline \multirow[t]{2}{*}{$\begin{array}{l}\text { Major Facilitator } \\
\text { Superfamily }\end{array}$} & B. longum infantis & Blon0915-F & CGTATTGGCTTTGTACGCATTT & 50 & 71 & {$[2]$} & 118 \\
\hline & & Blon0915-R & ATCGTGCCGGTGAGATTTAC & & & & \\
\hline \multirow[t]{2}{*}{$16 \mathrm{~S}$ rRNA } & $\begin{array}{l}\text { B. longum infantis, } \\
\text { B. longum longum, } \\
\text { B. indicum }\end{array}$ & Binf-F & CCATCTCTGGGATCGTCGG & 57 & & {$[31]$} & 562 \\
\hline & & Binf-R & TATCGGGGAGCAAGCGTGA & & & & \\
\hline \multirow[t]{2}{*}{$16 \mathrm{~S}$ rRNA } & B. infantis & HCBin-F & AGGATACGTTCGGCGTC & 60 & & [33] & 377 \\
\hline & & HCBin-R & CGCAAGATTCСTCTAGCA & & & & \\
\hline
\end{tabular}


Table 1. Cont

\begin{tabular}{|c|c|c|c|c|c|c|c|}
\hline Gene & Target Organism & Name & Sequence $\left(5^{\prime}-3^{\prime}\right)$ & $\begin{array}{l}\text { Annealing } \\
\text { Temp }\left({ }^{\circ} \mathrm{C}\right)\end{array}$ & $\%$ Coverage $^{1}$ & References & Length $(b p)^{2}$ \\
\hline 16S rRNA & Bifidobacterium & $\begin{array}{l}\text { g-Bif-F } \\
\text { g-Bif-R }\end{array}$ & $\begin{array}{l}\text { CTCCTGGAAACGGGTGG } \\
\text { GGTGTTCTTCCCGATATCTACA }\end{array}$ & 55 & & [31] & 550 \\
\hline 16S rRNA & All bacteria & $\begin{array}{l}\text { Uni-27F } \\
\text { Uni-1492R }\end{array}$ & $\begin{array}{l}\text { AGAGTTTGATCCTGGCTCAG } \\
\text { RGYTACCTTGTTACGACTT }\end{array}$ & 55 & & [34] & $\approx 1400$ \\
\hline
\end{tabular}

${ }^{1}$ Percent of exact alignment to 42 B. infantis strains. ${ }^{2}$ Amplicon size in base pairs. 
We chose four HMO-metabolizing genes that aligned $>97 \%$ to each $B$. infantis but not to any other bacteria and the included: exo- $\alpha$-sialidase gene (locus tag 'Blon2348'), glycoside-hydrolase gene (locus tag 'Blon2355'), haloacid dehalogenase-like hydrolase domain-containing protein gene (locus tag 'Blon2356') and $\beta$-lactamase domain protein gene (locus tag 'Blon2358'). To test the target specificity of the published primers and to design the new primers, $48 \mathrm{~B}$. longum group genomes and two $\mathrm{B}$. suis genomes were imported into Geneious (v R11.1.5), see Supplementary Materials Table S1. We aligned the genes from the selected genomes using MUSCLE (v 3.6) [35] alignment in Geneious and used them to design the primer pairs via Primer3 (v 2.3.7) [36]. Each primer pair was checked using NCBI primer-BLAST to ensure that it only matches $B$. infantis sequences [37]. All primers targeting HMO-metabolizing genes were checked against 387 genomes (Supplementary Materials Table S1) to assess their coverage (Table 1). Primers were obtained from Integrated DNA Technologies, Inc., (Coralville, IA, USA).

To ensure that the primers were adequate for targeted detection of $B$. infantis we used genomic DNA from isolated cultures of B. infantis (DSM 20088), B. longum (DSM 20219), B. breve (DSM 20213) and E. coli K12. The cultures were purchased from the German Collection of Microorganisms and Cell Cultures GmbH (DSMZ, Leibniz, Germany). The Bifidobacterium strains were incubated anaerobically at $37^{\circ} \mathrm{C}$ for $36 \mathrm{~h}$. The cells of each culture were centrifuged at 13,000 rpm for $1.5 \mathrm{~min}$ and washed in anaerobic PBS. Genomic DNA was then extracted using DNeasy PowerSoil Pro Kit (Qiagen, Germantown, MD, USA) following manufacturer's instructions. DNA concentrations were determined using an absorbance ratio of $260 / 280 \mathrm{~nm}$.

\subsection{Development of PCR Conditions}

PCR amplifications were performed using OneTaq Quick-Load 2X Master Mix (New England BioLabs, Ipswich, MA, USA), $200 \mathrm{nM}$ of each primer, and $10 \mathrm{ng}$ of template DNA ( $25 \mu \mathrm{L}$ total volume) following manufacturer's instructions. After the initial denaturation step at $94{ }^{\circ} \mathrm{C}$ for $30 \mathrm{~s}$, there were 30 cycles of $94{ }^{\circ} \mathrm{C}$ for $30 \mathrm{~s}$, the respective annealing temperature for $60 \mathrm{~s}$, and $72{ }^{\circ} \mathrm{C}$ for $60 \mathrm{~s}$, and then finally $68^{\circ} \mathrm{C}$ for $5 \mathrm{~min}$. The positive control was gDNA of isolated cultures of B. infantis (DSM 20088) or of B. breve (DSM 20213) and the negative control contained water instead of a template DNA were included with each batch.

\subsection{Confirmation of HMO-Metabolizing Genes in the Metagenomes by PCR}

To detect $B$. infantis by PCR in fecal samples, we used the $B$. infantis exo- $\alpha$-sialidase gene (locus tag 'Blon2348') primer pair (Sia-266F and Sia-676R). We also used 16S rRNA gene primers that target the genus Bifidobacterium (g-Bif-F and g-Bif-R) or universal 16S rRNA primers (27F and 1492R). All primers were purchased from Integrated DNA Technologies (IDT, Coralville, IA, USA).

\section{Results}

The first step in assessing the suitability of functionally differentiating B. infantis from other members of the $B$. longum group was to assess the phylogenetic relationships among members of this group. To accomplish this, we measured the average nucleotide identity (ANI) and shared gene content of all (387) publicly available B. longum group genomes (Supplemental Table S1). Four B. breve genomes (GCF_000158015.1, GCF_000213865.1, GCF_000220135.1, GCF_000226175.1) were included as an outgroup. Genomes in this analysis were stratified by the (sub)species in each pairwise comparison (Figure 1). As expected, genomes of the same species were the most similar, and within B. longum, subspecies were all $>95 \%$ similar to one another (Supplementary Materials Table S2). Subspecies clusters (B. longum, B. infantis, and B. suis) were clearly distinct by ANI, though substantially overlap in terms of shared gene content. By both metrics, B. longum and $B$. infantis were the most similar of the subspecies (Figure 1). Interestingly, each of these 
subspecies appears to separate into two groups by ANI. As expected, comparisons between the outgroup $B$. breve genomes and B. longum subspecies were the least similar.

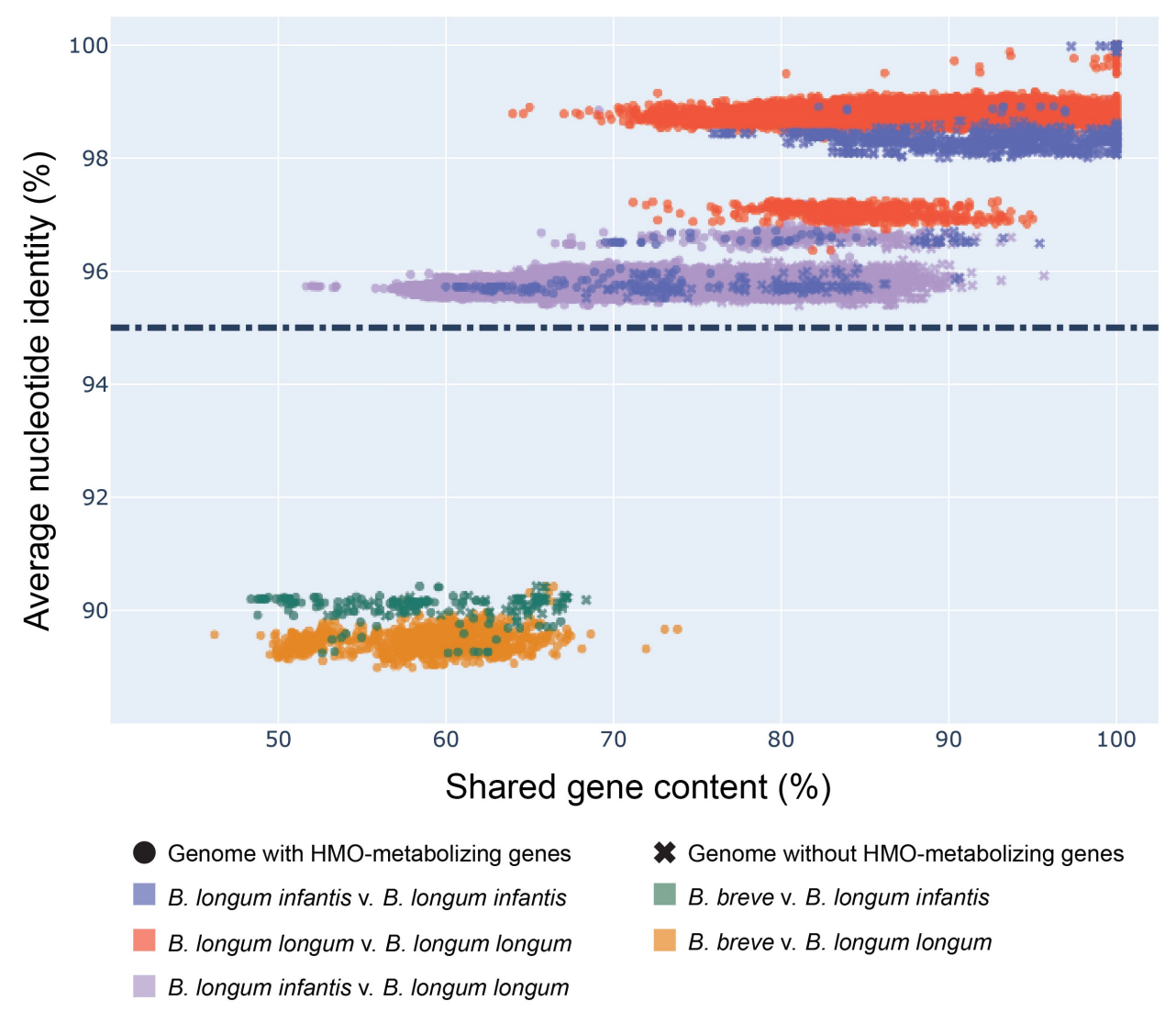

Figure 1. B. infantis genomes cluster separately from B.longum. Scatter plot of percent shared gene content versus ANI values across pairwise comparisons of Bifidobacterium genomes. Shared gene content calculated from BLAST and ANI values calculated by the Python package pyani. Presence of HMO-metabolizing genes determined by the occurrence of at least 60 of 79 (75\%) genes in a genome. Dashed line indicates the ANI cutoff score of $95 \%$ for genomes belonging to the same species.

Though $B$. infantis is resolvable from other B. longum subspecies by ANI, performing ANI calculations on metagenome assemblies is computationally expensive and requires high sequencing depth [38]. We therefore set out to determine whether individual genes might be suitable for distinguishing these subspecies. We explored the possibility that the presence of five genomic loci containing HMO-metabolizing genes could be used as a proxy for subspecies differences (Figure 2). To do so, we used BLAST to quantify the presence of the five subspecies-specific gene clusters within the same 387 Bifidobacterium genomes. Genomes previously characterized as B. infantis largely corresponded with both ANI-based clustering and with the presence of HMO-metabolizing genes, with 36 of $43 \mathrm{~B}$. infantislabeled genomes (Supplementary Materials Table S1), found in a distinct clade (Figure 2, top clade) and containing at least $93 \%$ of HMO-metabolizing genes. Crucially, seven out of these 43 B. infantis-labeled genomes, including 5 strains (CCUG 52486, CECT 7210, JCM 11660, KCTC 5934, 157F), did not contain most HMO-metabolizing genes, but also did not cluster with other $B$. infantis genomes based on ANI, suggesting that they may have been misclassified. These results persist when HMO-metabolizing genes were excluded from ANI calculations (Figure 2, Supplementary Materials Table S3), precluding the possibility that the ANI similarities are solely attributable to the presence or absence of those genes. Conversely, five unspecified B. longum genomes (GCF_900157085.1, GCF_900157065.1, GCF_900157185.1, GCF_900157075.1, GCF_902381625.1) clustered tightly with those from $B$. infantis strains and should, perhaps, be more specifically labeled as this particular 
subspecies (Figure 2). These genomes, although labeled as B. longum, contained HMOmetabolizing genes characteristic for $B$. infantis.

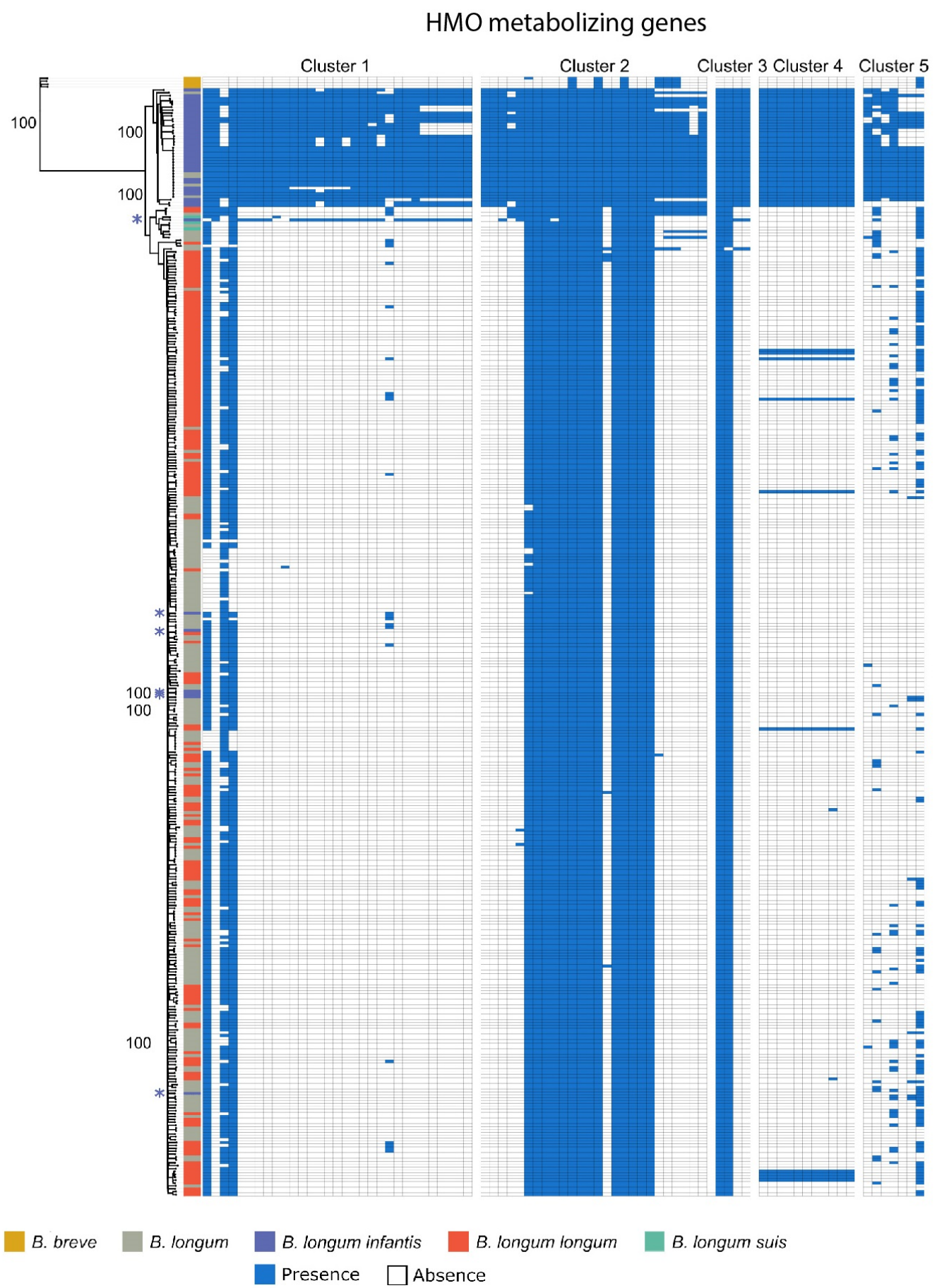

Figure 2. HMO-metabolizing gene clusters mirror phylogenetic separation of B. infantis. Rooted neighbor-joining tree of the average nucleotide identity (ANI) values of the same 387 Bifidobacterium genomes with HMO-metabolizing genes removed (Supplementary Materials Table S3). The tree is paired with a heatmap denoting the presence of HMO-metabolizing genes by BLASTN alignments (>95\% identity). Potentially misclassified $B$. infantis genomes noted with a purple asterisk.

The congruence of ANI and presence of HMO-metabolizing genes suggests that $B$. infantis diverged from other $B$. longum subspecies since the acquisition of these genes. To explore the history of the HMO-metabolizing gene clusters in more detail, we investigated 
their presence within and beyond the Bifidobacterium genus. We used BLAST to search for homologs of the 79 genes from the cluster against all available proteins in the NCBI database. Hits (see methods) for each of the HMO-metabolizing genes came largely from B. longum and B. breve genomes (Figure 3A, Supplementary Materials Table S6). Cluster 1 displayed the highest percentage of hits to genomes from both of these species at $64 \%$ and $24 \%$ respectively.

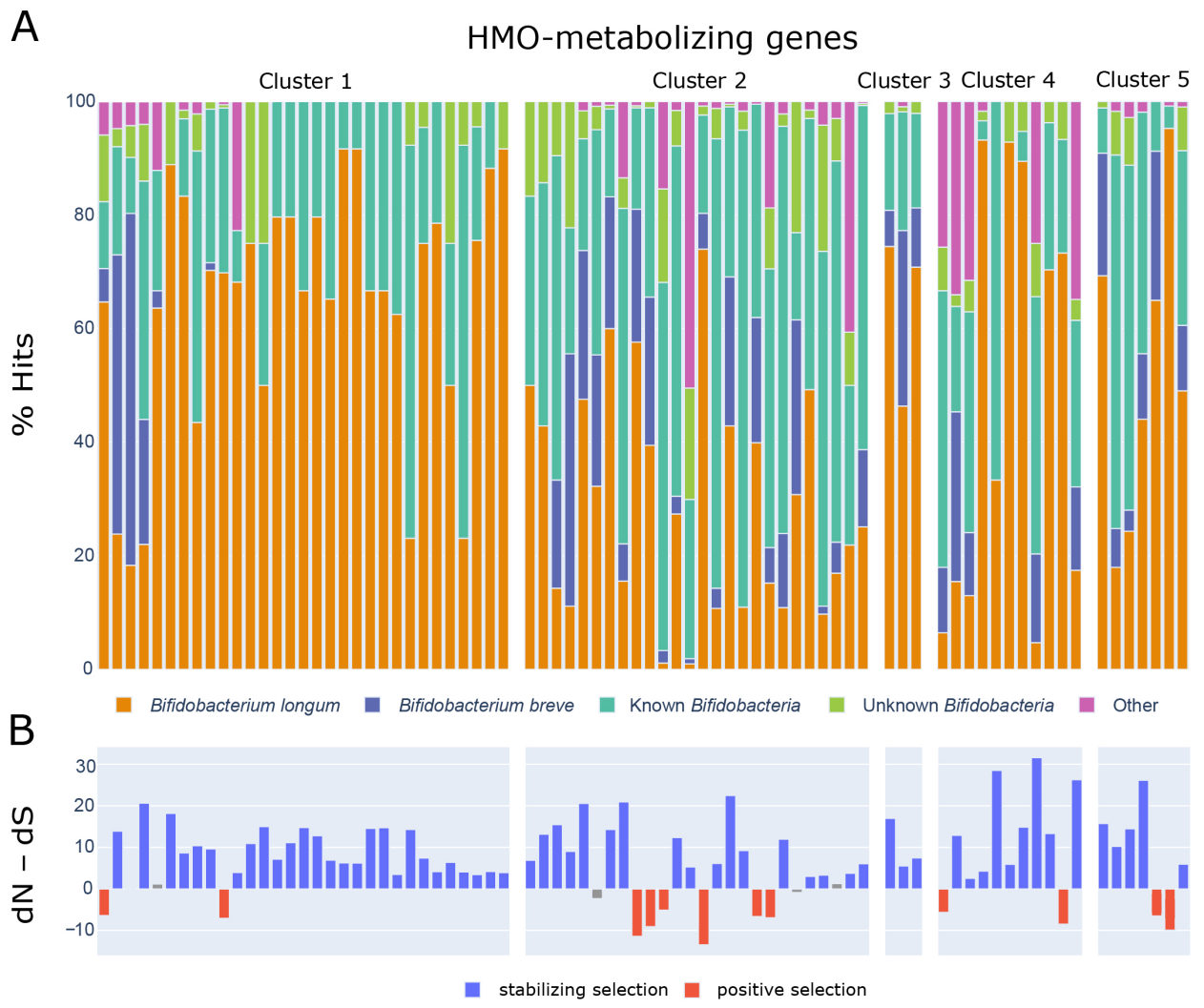

Figure 3. (A) Stacked bar plot showing taxonomic assignment of BLASTP hits (NCBI non-redundant database) for each HMO metabolizing gene. Known Bifidobacterium include any specified species in the Bifidobacterium genus other than B. longum or B. breve. Unknown Bifidobacterium include any unspecified Bifidobacterium taxa (Bifidobacterium, Bifidobacterium spp.). (B) Statistics from dN-dS analysis showing the probability and certainty that a given gene within the HMO-metabolizing gene clusters are under stabilizing or positive selection. Colored bars indicate statistical significance $(p<0.05)$. Analyses were conducted using the Nei-Gojobori method in MEGA X. All ambiguous positions were removed for each sequence pair (pairwise deletion option).

Because microbial genomes can be quite labile [39], we set out to assess whether the genes targeted by this approach were likely to remain stable by exploring the evolutionary pressures acting on the HMO use cassettes. To do so, we carried out systematic BLAST searches on each of the 78 protein-coding genes out of the $79 \mathrm{HMO}$-metabolizing genes. We hypothesized that genes under stabilizing selection would be better genomic markers of a subspecies, since these genes would be less likely to suffer gene loss or rapid mutation that would disrupt primer binding. We compared nucleotide differences in the substitution rates at nonsynonymous $(\mathrm{dN})$ and synonymous sites $(\mathrm{dS})$ using MEGA X [26,27]. A significant difference in the relative abundance of synonymous and nonsynonymous substitutions implies non-neutral selection, with a higher ratio of nonsynonymous substitutions indicating positive selection and a lower ratio indicating purifying selection [28]. Of the 78 protein-coding genes across the five clusters, 61 have undergone stabilizing selection (Supplementary Materials Table S7, Figure 3B). Twelve genes underwent positive selection and five did not show evidence of selection (neutral selection) (Figure 3B). 
After establishing that the five HMO-metabolizing gene clusters can distinguish $B$. infantis from the rest of the B. longum group members, we assessed the suitability of using $\mathrm{HMO}-$ metabolizing genes to generate primers specific to $B$. infantis. The development of the new primer sets was motivated by realizing that the existing primers to profile $B$. infantis were either too broad (e.g., 16S rRNA gene [33]) or too narrow (e.g., primers targeting sialidase gene [32]). Forty-six out of $79 \mathrm{HMO}-$ metabolizing genes covered were found to be in at least $85 \%$ of B. infantis strains. Two of these genes (Blon0218 and Blon0219) were found exclusively in the $B$. infantis clade (Figure 2, top clade) and might serve as potential future targets for primer development. Eleven additional genes (Blon2322, Blon2335, Blon2336, Blon2337, Blon2338, Blon2348, Blon2349, Blon2355, Blon0220, Blon0222, Blon0232) were present in all genomes within the $B$. infantis clade and one additional $B$. infantis genome (GCF_902167615.1) right outside the clade. This genome (GCF_902167615.1) contains a majority of HMO-metabolizing genes found in Cluster 1 (Figure 2) and would be classified as $B$. infantis by our analysis as well if it were limited to this HMO-metabolizing gene cluster.

Out of the 11 genes, we selected four genes (Blon2348, Blon2355, Blon2356 and Blon2358) that aligned well with the clade of 41 genomes we consider to be $B$. infantis (Supplementary Materials Table S1). Genes exo- $\alpha$-sialidase (Blon2348) and glycosidehydrolase (Blon2355) were present in all genomes within the clade and one additional B. infantis genome (GCF_902167615.1) right outside of it. We also selected genes Blon2356 and Blon2358 that were present in $34(83 \%)$ of the genomes within this clade and the additional B. infantis genome (GCF_902167615.1) as well. All four genes also had conserved regions among the $B$. infantis, making them good candidates for primer development.

Primer pairs for these four genes were tested in silico against the 387 downloaded genomes (Table 1, Supplementary Materials Table S1). The primer specificity to the $B$. infantis clade matched clustering of strains based on ANI (Figure 2). The same six B. infantis-labeled genomes that failed to cluster with B. infantis by ANI provided no hits to our primer pairs (Supplementary Materials Table S1), providing additional evidence that these $B$. infantis-named strains may have been misclassified. Similarly, the five unspecified B. longum genomes (GCF_900157085.1, GCF_900157065.1, GCF_900157185.1, GCF_900157075.1,GCF_902381625.1) that were clustered within the B. infantis clade (Figure 2) perfectly hybridized in silico to our primer sets.

To confirm the specificity of the designed primer pairs in vitro, PCR assays were performed with three Bifidobacterium reference strains, an E. coli K12 strain and a negative control. The amplification product was again specific to $B$. infantis (Table 1 , the first 4 primer pairs). All four primers aligned $100 \%$ to $B$. infantis and not to any other bacteria. These primers were then applied to verify $B$. infantis presence in sequenced fecal samples.

Finally, we used the five HMO-metabolizing gene clusters to distinguish $B$. infantis from the rest of the $B$. longum subspecies in the gut microbiomes of human children using fecal samples in the RESONANCE cohort of young, healthy children [40]. We applied the specificity of this HMO-metabolizing cluster to identify B. infantis from metagenomic analysis of 1092 fecal samples. These samples included pregnant mothers, and children ranging from infants to adolescents. Sequences belonging to the Bifidobacterium longum species were also found in the metagenomes of most (158 of 203 or $78 \%$ ) of the samples from children under two years old analyzed. By searching for the HMO-metabolizing gene clusters in shotgun metagenomic data, we observed that 15 of $203(7.4 \%)$ samples from children under the age of two harbor $B$. infantis (Figure 4). The gut microbiomes of three of the subjects were sampled at later times and no longer contained $B$. infantis, suggesting a decrease in abundance around one year of age. PCR amplification of one HMO-metabolizing gene (the sialidase gene Blon2348) in a subset of samples corroborated the metagenomic evidence (Figure 4 ). When screened by PCR with $B$. infantis-specific primers, only 13 of $162(8.0 \%)$ samples taken from children under two and 4 of $411(1.0 \%)$ samples taken from mothers were found to have B. infantis. 


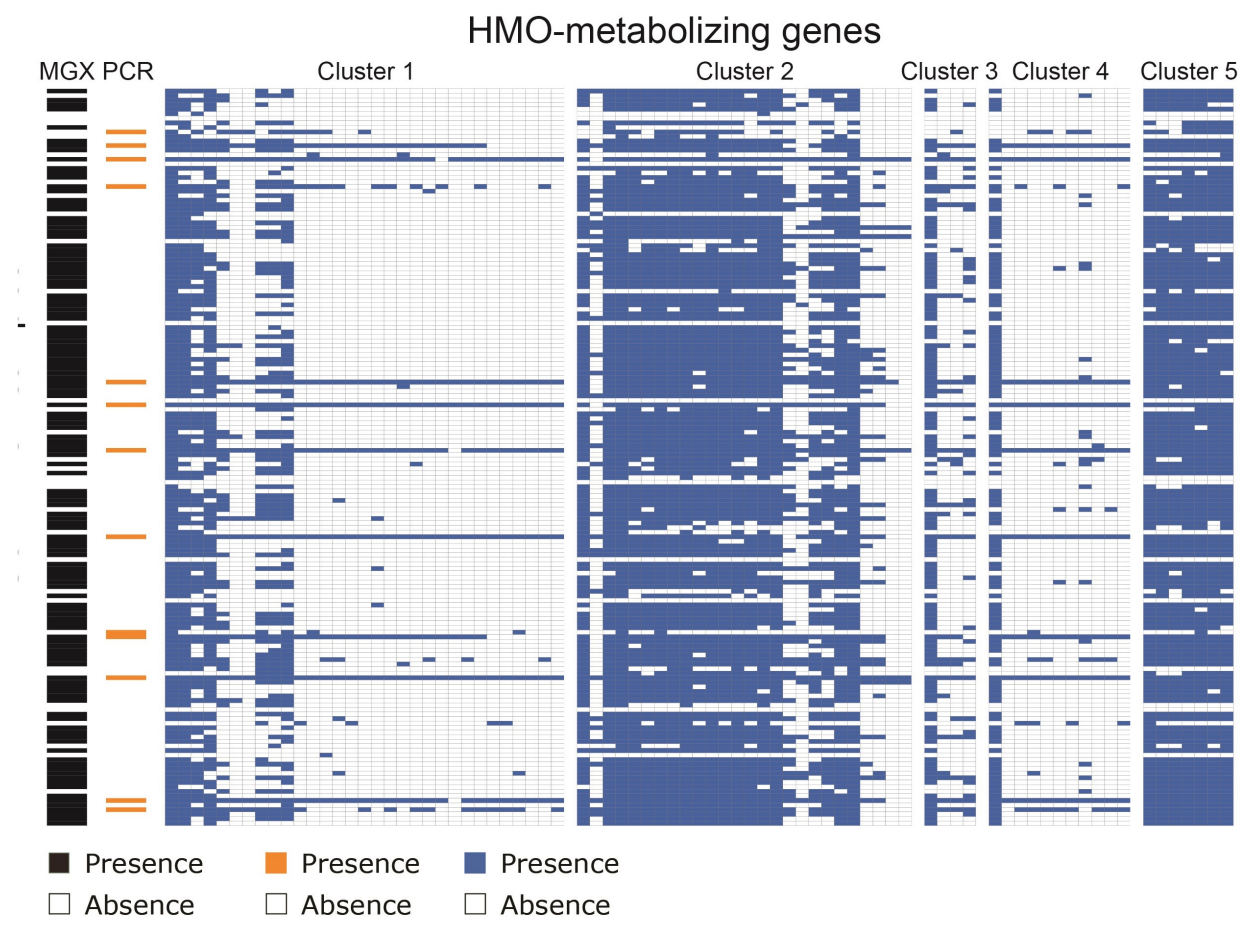

Figure 4. B. infantis presence observed in 15 of 203 (7.4\%) samples from children under the age of two via PCR or metagenomics. Results from tests on samples from children under the age of two for the presence of HMO-metabolizing genes either via metagenomics $(n=203)$ or PCR $(n=162)$. The presence of $B$. infantis in metagenomes $(n=15)$ was determined by mapped Bowtie 2 alignments to HMO-metabolizing genes. The PCR presence of $B$. infantis $(n=13)$ was determined by PCR using primers designed to target the gene Blon2348 unique to the subspecies, with presence noted in orange and absence in white. Presence of Bifidobacterium longum calculated by MetaPhlAn is also noted on the left-hand side in the MGX column in black and absence in white.

\section{Discussion}

The human gut microbiome, though better characterized than most microbial communities, remains poorly understood. This is especially true in very young children, whose gut microbial communities are seeded at birth, shaped profoundly by the method of their birth and the nature of their diet, and in constant flux. The identification nearly 100 years ago of a component of human breast milk (HMO) that appears to have evolved solely to feed the developing microbiomes of infants hints at the critical importance of microbiome development as part of overall child development [41]. Here, we have shown that by focusing on the functional differences between microbial subspecies, in particular those differences that provide a critical metabolic function such as HMO use, we can increase taxonomic resolution through culture-independent means.

The Bifidobacterium genus is incredibly common in children's guts. In this study, $96 \%$ of children have them as confirmed by PCR assay and metagenomic search and these findings are consistent with other early life gut microbiome studies [42,43]. Within the Bifidobacterium genus, many species harbor genes that allow them to make use of complex polysaccharides. Yet, there are important functional differences between members of this genus, and even, as we have shown here, between the subspecies B. longum and $B$. infantis. This is not uncommon; a recent study showed an average of $13 \%$ (and as much as $25 \%$ ) difference in gene content between common gut microbiome species from different individuals [39]. Recent comparisons of the infant gut microbiome indicate that $B$. infantis is more prevalent among infants from countries with limited resources such as Gambia (76.9\%) and Bangladesh, and less prevalent in more developed countries such as Finland $(10 \%)$, with increasing prevalence in countries such as Estonia (20\%) 
and Russia (23\%) [44,45]. Using metagenomic sequencing and a gene function-centered approach allowed us to show that only $7.4 \%$ children under 2 years old in a cohort of US children harbored the subspecies $B$. infantis, and we showed that this is synonymous with harboring five HMO-metabolizing gene clusters. These results are on par with a recent study on microbial dysbiosis in American infants in the first six months of life that showed remarkably low prevalence of HMO-metabolizing capacity of $10 \%$ that accompanied other indicators of unhealthy microbiomes [46]. The lower prevalence of $B$. infantis in our cohort is likely due to inclusion of children who have transitioned from the diet of human milk to solid foods. Given the apparent decline of $B$. infantis in western populations, and the beneficial associations of $B$. infantis with allergies and autoinflammatory disorders [47,48], other health conditions [49], and healthy brain development [50], it is critical to have accurate classification methods for this microbe to improve our ability to understand the links between HMO use and early child development.

This study addresses some of the existing limitations for studying B. infantis. Although amplicon (16S rRNA gene) sequencing is typically only capable of resolving genus-level differences between microbes, both PCR and shotgun metagenomic sequencing have the capacity to interrogate gene-level variation. Yet these two methods have important tradeoffs; PCR-based detection is straightforward, cost-effective, and able to detect very rare gene functions, but difficult to scale to large numbers of samples or to identify many gene functions at once. Shotgun metagenomic sequencing can be readily scaled, but is substantially more costly, especially at sequencing depths necessary to detect lowabundance genes. Here, we showed that metagenomic sequencing largely agrees with PCR-based amplification, at least for one HMO-metabolizing gene. A custom primer pair designed to target the HMO-metabolizing sialidase gene, Blon2348, in B. infantis was used in PCR in this study. Eleven of the 79 HMO-metabolizing genes were also found in all genomes within the B. infantis ANI cluster; a subset of these might be used for future primer development. Further using PCR to investigate the presence of many genes in multiple gene clusters, however, would have been impractical, and the concordance of dozens of genes using metagenomic sequencing lends increased credence to the presence of these genes in the samples identified through this method.

We were able to identify six genomes that were previously identified as $B$. infantis that should in fact be classified as $B$. longum, as well as five genomes that do not have subspecies resolution in Genbank that likely should be classified as B. infantis. The high concordance between average nucleotide identity and the presence of all $5 \mathrm{HMO}$ use clusters suggests that acquisition or loss of these genes occurred in a single common ancestor and continued divergence with positive selection due to occupying the HMO use niche. However, this need not always be the case; horizontal gene transfer (HGT) can lead to gene function sharing across species boundaries [51,52]. In human microbiome epidemiology, what likely matters in shaping health outcomes is functional variation, for which taxonomic profiling is an imperfect proxy. We believe that a function-based metagenomic approach is essential for untangling the complexity of the gut microbiome and its relationship to human health.

Supplementary Materials: The following are available at https:/ / www.mdpi.com/article/10.3390/ nu13082833/s1, Table S1: Bifidobacterium genomes used in this study. Table S2: Average nucleotide identity between two genomes. Table S3: Average nucleotide identity between two genomes with HMO-metabolizing genes removed. Table S4: The composition of the cohort used in this study. Table S5: Fecal sample IDs used in this study. Table S6: Composition of BLASTP alignments of HMO-metabolizing genes to taxa contained in NCBI's non-redundant database. Table S7: Statistics from $\mathrm{dN}-\mathrm{dS}$ analysis of $\mathrm{HMO}-$ metabolizing genes.

Author Contributions: L.T., K.S.B. and V.K.-C. designed the study; S.R. and A.F. processed the samples; L.T. and K.S.B. designed and wrote the code and analyzed the data; L.T., K.S.B. and V.K.-C. wrote, edited, and finalized the manuscript. All authors have read and agreed to the published version of the manuscript. 
Funding: This research was funded by the NIH UG3 OD023313 (V.K.C.) and the American Society for Microbiology Undergraduate Research Fellowship (L.T.).

Institutional Review Board Statement: The study was conducted according to the guidelines of the Declaration of Helsinki, and approved by the Institutional Review Board Rhode Island Hospital, Providence, RI, USA (protocol codes 1501119-13 and 1501119-14, date of most recent approval: 24 January 2021).

Informed Consent Statement: Informed assent or consent was obtained from all subjects involved in the study.

Data Availability Statement: Metagenome data were deposited in NCBI's Sequence Read Archive (SRA), under BioProject PRJNA695570 and accession numbers SAMN17618458-9435. Code for data analysis, statistics, and figures generated for this manuscript is available on OSF.io [40].

Acknowledgments: The samples were collected as a part of an ongoing NIH ECHO study done by RESONANCE consortium and we thank families participating in the RESONANCE cohort. We thank Libusha Kelly and Sarah Comstock for reviewing the manuscript and providing helpful comments. We thank Ivica Ceraj for help with data analysis.

Conflicts of Interest: The authors declare no conflict of interest. The funders had no role in the design of the study; in the collection, analyses, or interpretation of data; in the writing of the manuscript, or in the decision to publish the results.

\section{Abbreviations \\ The following abbreviations are used in this manuscript: \\ HMO Human Milk Oligossacharide}

\section{References}

1. Lebreton, F.; Manson, A.L.; Saavedra, J.T.; Straub, T.J.; Earl, A.M.; Gilmore, M.S. Tracing the enterococci from paleozoic origins to the hospital. Cell 2017, 169, 849-861.e13. [CrossRef] [PubMed]

2. Frese, S.A.; Hutton, A.A.; Contreras, L.N.; Shaw, C.A.; Palumbo, M.C.; Casaburi, G.; Xu, G.; Davis, J.C.; Lebrilla, C.B.; Henrick, B.M.; et al. Persistence of supplemented Bifidobacterium longum subsp. infantis EVC001 in breastfed infants. mSphere 2017, 2, e00501-17. [CrossRef] [PubMed]

3. Le Doare, K.; Holder, B.; Bassett, A.; Pannaraj, P.S. Mother's milk: A purposeful contribution to the development of the infant microbiota and immunity. Front. Immunol. 2018, 9, 361. [CrossRef] [PubMed]

4. Milani, C.; Lugli, G.A.; Duranti, S.; Turroni, F.; Bottacini, F.; Mangifesta, M.; Sanchez, B.; Viappiani, A.; Mancabelli, L.; Taminiau, B.; et al. Genomic encyclopedia of type strains of the genus Bifidobacterium. Appl. Environ. Microbiol. 2014, 80, 6290-6302. [CrossRef] [PubMed]

5. Milani, C.; Turroni, F.; Duranti, S.; Lugli, G.A.; Mancabelli, L.; Ferrario, C.; van Sinderen, D.; Ventura, M. Genomics of the genus Bifidobacterium reveals species-specific adaptation to the glycan-rich gut environment. Appl. Environ. Microbiol. 2016, 82, 980-991. [CrossRef] [PubMed]

6. Sun, Z.; Zhang, W.; Guo, C.; Yang, X.; Liu, W.; Wu, Y.; Song, Y.; Kwok, L.Y.; Cui, Y.; Menghe, B.; et al. Comparative genomic analysis of 45 type strains of the genus Bifidobacterium: A snapshot of its genetic diversity and evolution. PLoS ONE 2015, 10, e0117912. [CrossRef]

7. Garrido, D.; Kim, J.H.; German, J.B.; Raybould, H.E.; Mills, D.A. Oligosaccharide binding proteins from Bifidobacterium longum subsp. infantis reveal a preference for host glycans. PLoS ONE 2011, 6, e17315. [CrossRef]

8. LoCascio, R.G.; Desai, P.; Sela, D.A.; Weimer, B.; Mills, D.A. Broad conservation of milk utilization genes in Bifidobacterium longum subsp. infantis as revealed by comparative genomic hybridization. Appl. Environ. Microbiol. 2010, 76, 7373-7381. [CrossRef]

9. Sela, D.A.; Chapman, J.; Adeuya, A.; Kim, J.H.; Chen, F.; Whitehead, T.R.; Lapidus, A.; Rokhsar, D.S.; Lebrilla, C.B.; German, J.B.; et al. The genome sequence of Bifidobacterium longum subsp. infantis reveals adaptations for milk utilization within the infant microbiome. Proc. Natl. Acad. Sci. USA 2008, 105, 18964-18969. [CrossRef]

10. Satokari, R.M.; Vaughan, E.E.; Favier, C.F.; Doré, J.; Edwards, C.; Vos, W.M.d. Diversity of Bifidobacterium and Lactobacillus spp. in breast-fed and formula-fed infants as assessed by $16 \mathrm{~S}$ rDNA sequence differences. Microb. Ecol. Health Dis. 2002, 14, 97-105. [CrossRef]

11. Bonjoch, X.; Balleste, E.; Blanch, A.R. Multiplex PCR with 16S rRNA gene-targeted primers of Bifidobacterium spp. to identify sources of fecal pollution. Appl. Environ. Microbiol. 2004, 70, 6. [CrossRef]

12. Matsuki, T.; Watanabe, K.; Fujimoto, J.; Kado, Y.; Takada, T.; Matsumoto, K.; Tanaka, R. Quantitative PCR with 16S rRNA-genetargeted species-specific primers for analysis of human intestinal Bifidobacteria. Appl. Environ. Microbiol. 2004, 70, 167-173. [CrossRef] 
13. Johnson, J.S.; Spakowicz, D.J.; Hong, B.Y.; Petersen, L.M.; Demkowicz, P.; Chen, L.; Leopold, S.R.; Hanson, B.M.; Agresta, H.O.; Gerstein, M.; et al. Evaluation of $16 \mathrm{~S}$ rRNA gene sequencing for species and strain-level microbiome analysis. Nat. Commun. 2019, 10, 1-11. [CrossRef]

14. Truong, D.T.; Franzosa, E.A.; Tickle, T.L.; Scholz, M.; Weingart, G.; Pasolli, E.; Tett, A.; Huttenhower, C.; Segata, N. MetaPhlAn2 for enhanced metagenomic taxonomic profiling. Nat. Methods 2015, 12, 902-903. [CrossRef]

15. Wood, D.E.; Lu, J.; Langmead, B. Improved metagenomic analysis with Kraken 2. Genome Biol. 2019, 20, 257. [CrossRef]

16. Marcobal, A.; Barboza, M.; Froehlich, J.W.; Block, D.E.; German, J.B.; Lebrilla, C.B.; Mills, D.A. Consumption of human milk oligosaccharides by gut-related microbes. J. Agric. Food Chem. 2010, 58, 5334-5340. [CrossRef]

17. Marcobal, A.; Barboza, M.; Sonnenburg, E.; Pudlo, N.; Martens, E.; Desai, P.; Lebrilla, C.; Weimer, B.; Mills, D.; German, J.B.; et al. Bacteroides in the infant gut consume milk oligosaccharides via mucus-utilization pathways. Cell Host Microbe 2011, 10, 507-514. [CrossRef]

18. Ruiz-Moyano, S.; Totten, S.M.; Garrido, D.A.; Smilowitz, J.T.; German, J.B.; Lebrilla, C.B.; Mills, D.A. Variation in consumption of human milk oligosaccharides by infant gut-associated strains of Bifidobacterium breve. Appl. Environ. Microbiol. 2013, 79, 6040-6049. [CrossRef]

19. Yu, Z.T.; Chen, C.; Newburg, D.S. Utilization of major fucosylated and sialylated human milk oligosaccharides by isolated human gut microbes. Glycobiology 2013, 23, 1281-1292. [CrossRef]

20. Garrido, D.; Ruiz-Moyano, S.; Lemay, D.G.; Sela, D.A.; German, J.B.; Mills, D.A. Comparative transcriptomics reveals key differences in the response to milk oligosaccharides of infant gut-associated bifidobacteria. Sci. Rep. 2015, 5, 13517. [CrossRef]

21. Thongaram, T.; Hoeflinger, J.L.; Chow, J.; Miller, M.J. Human milk oligosaccharide consumption by probiotic and humanassociated bifidobacteria and lactobacilli. J. Dairy Sci. 2017, 100, 7825-7833. [CrossRef] [PubMed]

22. Konstantinidis, K.T.; Tiedje, J.M. Genomic insights that advance the species definition for prokaryotes. Proc. Natl. Acad. Sci. USA 2005, 102, 2567-2572. [CrossRef] [PubMed]

23. Pritchard, L.; Glover, R.H.; Humphris, S.; Elphinstone, J.G.; Toth, I.K. Genomics and taxonomy in diagnostics for food security: soft-rotting enterobacterial plant pathogens. Anal. Methods 2016, 8, 12-24. [CrossRef]

24. Kurtz, S.; Phillippy, A.; Delcher, A.L.; Smoot, M.; Shumway, M.; Antonescu, C.; Salzberg, S.L. Versatile and open software for comparing large genomes. Genome Biol. 2004, 5, 1-9. [CrossRef] [PubMed]

25. Altschul, S.F.; Gish, W.; Miller, W.; Myers, E.W.; Lipman, D.J. Basic local alignment search tool. J. Mol. Biol. 1990, $215,403-410$. [CrossRef]

26. Kumar, S.; Stecher, G.; Li, M.; Knyaz, C.; Tamura, K. MEGA X: Molecular evolutionary genetics analysis across computing platforms. Mol. Biol. Evol. 2018, 35, 1547-1549. [CrossRef]

27. Stecher, G.; Tamura, K.; Kumar, S. Molecular evolutionary genetics analysis (MEGA) for macOS. Mol. Biol. Evol. 2020, 37, 1237-1239. [CrossRef]

28. Kryazhimskiy, S.; Plotkin, J.B. The population genetics of dN/dS. PLoS Genet. 2008, 4, e1000304. [CrossRef]

29. McIver, L.J.; Abu-Ali, G.; Franzosa, E.A.; Schwager, R.; Morgan, X.C.; Waldron, L.; Segata, N.; Huttenhower, C. bioBakery: A meta'omic analysis environment. Bioinformatics 2018, 34, 1235-1237. [CrossRef]

30. Langmead, B.; Wilks, C.; Antonescu, V.; Charles, R. Scaling read aligners to hundreds of threads on general-purpose processors. Bioinformatics 2019, 35, 421-432. [CrossRef]

31. Tannock, G.W.; Lawley, B.; Munro, K.; Gowri Pathmanathan, S.; Zhou, S.J.; Makrides, M.; Gibson, R.A.; Sullivan, T.; Prosser, C.G.; Lowry, D.; et al. Comparison of the Compositions of the stool microbiotas of infants fed goat milk formula, cow milk-based formula, or breast milk. Appl. Environ. Microbiol. 2013, 79, 3040-3048. [CrossRef]

32. Lawley, B.; Munro, K.; Hughes, A.; Hodgkinson, A.J.; Prosser, C.G.; Lowry, D.; Zhou, S.J.; Makrides, M.; Gibson, R.A.; Lay, C.; et al Differentiation of Bifidobacterium longum subspecies longum and infantis by quantitative PCR using functional gene targets. PeerJ 2017, 5, e3375. [CrossRef]

33. Hong, W.S.; Chen, M.J. Rapid identification of Bifidobacteria in dairy products by gene-targeted species-specific PCR technique and DGGE. Asian-Australas. J. Anim. Sci. 2007, 20, 1887-1894. [CrossRef]

34. Weisburg, W.G.; Barns, S.M.; Pelletier, D.A.; Lane, D.J. 16 S ribosomal DNA amplification for phylogenetic study J. Bacteriol. 1996 173, 697-703 [CrossRef]

35. Edgar, R.C. MUSCLE: Multiple sequence alignment with high accuracy and high throughput. Nucleic Acids Res. 2004, 32, 1792-1797. [CrossRef]

36. Untergasser, A.; Nijveen, H.; Rao, X.; Bisseling, T.; Geurts, R.; Leunissen, J.A. Primer3Plus, an enhanced web interface to Primer3. Nucleic Acids Res. 2007, 35, W71-W74. [CrossRef]

37. Ye, J.; Coulouris, G.; Zaretskaya, I.; Cutcutache, I.; Rozen, S.; Madden, T.L. Primer-BLAST: A tool to design target-specific primers for polymerase chain reaction. BMC Bioinform. 2012, 13, 134. [CrossRef]

38. Jain, C.; Rodriguez, R.L.M.; Phillippy, A.M.; Konstantinidis, K.T.; Aluru, S. High throughput ANI analysis of 90K prokaryotic genomes reveals clear species boundaries. Nat. Commun. 2018, 9, 5114. [CrossRef]

39. Zhu, A.; Sunagawa, S.; Mende, D.R.; Bork, P. Inter-individual differences in the gene content of human gut bacterial species. Genome Biol. 2015, 16, 82. [CrossRef] 
40. Bonham, K.S.; Bruchhage, M.M.K.; Rowland, S.; Volpe, A.R.; Dyer, K.; Consortium, R.; D’Sa, V.; Huttenhower, C.; Deoni, S.C.L.; Klepac-Ceraj, V. Gut microbes and their genes are associated with brain development and cognitive function in healthy children. bioRxiv 2020. [CrossRef]

41. Kunz, C. Historical aspects of human milk oligosaccharides. Adv. Nutr. 2012, 3, 430S-439S. [CrossRef] [PubMed]

42. Yatsunenko, T.; Rey, F.E.; Manary, M.J.; Trehan, I.; Dominguez-Bello, M.G.; Contreras, M.; Magris, M.; Hidalgo, G.; Baldassano, R.N.; Anokhin, A.P.; et al. Human gut microbiome viewed across age and geography. Nature 2012, 486, 222-227. [CrossRef] [PubMed]

43. Bäckhed, F.; Roswall, J.; Peng, Y.; Feng, Q.; Jia, H.; Kovatcheva-Datchary, P.; Li, Y.; Xia, Y.; Xie, H.; Zhong, H.; et al. Dynamics and stabilization of the human gut microbiome during the first year of life. Cell Host Microbe 2015, 17, 690-703. [CrossRef] [PubMed]

44. Davis, J.C.C.; Lewis, Z.T.; Krishnan, S.; Bernstein, R.M.; Moore, S.E.; Prentice, A.M.; Mills, D.A.; Lebrilla, C.B.; Zivkovic, A.M. Growth and morbidity of Gambian infants are influenced by maternal milk oligosaccharides and infant gut microbiota. Sci. Rep. 2017, 7, 40466. [CrossRef]

45. Vatanen, T.; Plichta, D.R.; Somani, J.; Münch, P.C.; Arthur, T.D.; Hall, A.B.; Rudolf, S.; Oakeley, E.J.; Ke, X.; Young, R.A.; et al. Genomic variation and strain-specific functional adaptation in the human gut microbiome during early life. Nat. Microbiol. 2019, 4, 470-479. [CrossRef]

46. Casaburi, G.; Duar, R.M.; Brown, H.; Mitchell, R.D.; Kazi, S.; Chew, S.; Cagney, O.; Flannery, R.L.; Sylvester, K.G.; Frese, S.A.; et al. Metagenomic insights of the infant microbiome community structure and function across multiple sites in the United States. Sci. Rep. 2021, 11, 1472. [CrossRef]

47. Fu, L.; Song, J.; Wang, C.; Fu, S.; Wang, Y. Bifidobacterium infantis potentially alleviates shrimp tropomyosin-induced allergy by tolerogenic dendritic cell-dependent induction of regulatory T cells and alterations in gut microbiota. Front. Immunol. 2017, 8, 1536. [CrossRef]

48. Wang, W.; Luo, X.; Zhang, Q.; He, X.; Zhang, Z.; Wang, X. Bifidobacterium infantis relieves allergic asthma in mice by regulating Th1/Th2. Med. Sci. Monit. 2020, 26, e920583. [CrossRef]

49. Navarro-Tapia, E.; Sebastiani, G.; Sailer, S.; Toledano, L.A.; Serra-Delgado, M.; García-Algar, Ó.; Andreu-Fernández, V. Probiotic supplementation during the perinatal and infant period: Effects on gut dysbiosis and disease. Nutrients 2020, 12, 2243. [CrossRef]

50. Cerdó, T.; Ruíz, A.; Suárez, A.; Campoy, C. Probiotic, Prebiotic, and Brain Development. Nutrients 2017, 9, 1247. [CrossRef]

51. Smillie, C.S.; Smith, M.B.; Friedman, J.; Cordero, O.X.; David, L.A.; Alm, E.J. Ecology drives a global network of gene exchange connecting the human microbiome. Nature 2011, 480, 241-244. [CrossRef]

52. Brito, I.L.; Yilmaz, S.; Huang, K.; Xu, L.; Jupiter, S.D.; Jenkins, A.P.; Naisilisili, W.; Tamminen, M.; Smillie, C.S.; Wortman, J.R.; et al. Mobile genes in the human microbiome are structured from global to individual scales Nature 2016, 535, 435-439. [CrossRef] 